\title{
Stability and electronic properties of carbon nanotubes doped with transition metal impurities
}

\author{
S. Azevedo ${ }^{1, a}$, C. Chesman ${ }^{2}$, and J.R. Kaschny ${ }^{3}$ \\ 1 Departamento de Física, Universidade Estadual de Feira de Santana, KM-03, BR-116, 44031-460 Feira de Santana - BA, \\ Brazil \\ 2 Departamento de Física Teórica e Experimental, Universidade Federal do Rio Grande do Norte, 59078-970 Natal - RN, Brazil \\ 3 Coordenação de Engenharia Elétrica, Instituto Federal da Bahia, Av. Amazonas 3150, 45030-220 Vitória da Conquista - BA, \\ Brazil
}

Received 24 July 2009 / Received in final form 2 November 2009

Published online 23 February 2010 - (c) EDP Sciences, Società Italiana di Fisica, Springer-Verlag 2010

\begin{abstract}
We apply first-principles method to investigate the effect of the diameter on the stability and electronic properties of zigzag carbon nanotubes doped with iron, nickel and manganese impurity atoms. In this contribution we follow the evolution of the electronic and structural properties as a function of the nanotube diameter. As a general result, we found that the binding energy decreases with the increasing nanotube radius. Additionally, depending on the interaction of transition metal impurity with the tubular carbon structure, it is observed that the total magnetization varies with the tube diameter due to hybridization and confinement effects. It is also shown that such magnetization varies with the curvature radius, increasing for manganese impurity atoms and decreasing for iron and nickel.
\end{abstract}

\section{Introduction}

Physical properties of materials at the nanoscale size can differ dramatically from their bulk counterparts. This is especially appreciable when the electronic properties of nanomaterials are analyzed. Since the quantum behavior of electrons on nanometric scale becomes evident, such properties turn to be very sensitive to the size, shape, and symmetry of the atomic arrangement.

Curved nanoscale structures, which the best known examples are carbon fullerenes and nanotubes, have been the focus of increasing scientific and technological interest, due to their unique electronic and mechanical properties [1-3]. Single-walled carbon nanotubes (SWCNTs) were first discovered by arc discharge, with a carbon rod electrode containing transition metal (TM) catalysts. Thereafter, substantial progress has been made to synthesize carbon nanotubes using, for example, laser-ablation and chemical vapor deposition. A common characteristic of these methods is the requirement of a metal or a metal compound catalyst, in particular, 3d-TMs such as $\mathrm{Fe}, \mathrm{Co}$, and Ni. These metals can eventually be mixed with the carbon material, introducing metal impurities in the nanostructure under synthesis, affecting the electronic and magnetic properties of the resulting nanotube. Therefore, the investigation of TM atoms interactions with SWCNTs should be of great interest in nanotechnology. Several theoretical $[4,5]$ and experimental $[6,7]$ work, about

\footnotetext{
a e-mail: sazevedo@uefs.br
}

the influence of TM impurities on the properties of SWCNTs and about transition metal adsorption in graphite, have been reported in the literature. Recently, iron filled SWCNTs [8-10] and the absorption of Fe clusters by carbon nanotubes [11] have been the subject of many studies, with potential applications on the development of spintronic devices. Nevertheless, the complete understanding of the effects of such impurities remains a challenging issue. For example, to our knowledge, the influence of the tube diameter on the stability and magnetic properties of SWCNTs, containing Fe, remains an open question.

Taking into account the technological interest on the development of field emission devices, the work function is an important parameter to characterize the electronic structure of the SWCNTs. Suzuki et al., using ultraviolet photoemission spectroscopy (UPS), have measured such work function, obtaining a value of about $4.8 \mathrm{eV}[12]$. In other experimental report, Masashi and coworker, using photoelectron emission (PEE), have obtained a value of $4.95 \mathrm{eV}$ [13]. More recently, Hyun et al., have developed an experimental method to control the diameter of carbon nanotubes and to investigate the effect of the tube diameter on the field emission properties [14]. They observe that decreasing the tube diameter increases the field emission effect, while the emission stability remains almost unaffected by the tube length and radius.

In the present contribution, we present a systematic investigation about the influence of transition metal impurities on the work function, electronic structure and stability 
of carbon nanotubes, using first-principles method. Additionally, we investigate how the electronic structure and the stability of carbon nanotubes are affected by the impurity as a function of the tube diameter.

\section{Calculation procedure}

In the present work we apply the density functional theory (DFT), including spin polarization (SP), as implemented in the SIESTA code [15-17]. The calculations are performed using the generalized gradient approximation (GGA), for the exchange-correlation functional [18], and norm-conserving Troullier-Martins pseudopotentials, in the Kleinmann-Bylander factorized form [19-21]. For the basis set, we use a double- $\zeta$ basis composed of numerical atomic orbitals of finite range including polarization function. For comparison purposes all calculations were also performed without spin polarization (SUP). The initial structure incorporates one metal impurity atom, initially placed in the central position of the tube supercell. We use periodic-boundary conditions and a supercell approximation, with $64 \mathrm{C}$ atom plus one atom $-\mathrm{Fe}, \mathrm{Ni}$, or Mn, with a resulting length of $8.52 \AA$. Additionally, the lateral separation of $15 \AA$ between tube axis prevents any tube-tube interaction. Such cell size should be enough to ensure that impurity atoms, in neighboring cells, do not interact with each other. Finally, the atomic arrangement was considered to be optimized when the remaining forces were smaller than $0.1 \mathrm{eV} / \AA$.

\section{Structural properties}

In the present calculations, we considered $(6,0),(7,0)$, $(8,0),(9,0)$, and $(12,0)$ SWCNTs doped with Fe, Mn, and Ni impurity atoms. For the initial position of the impurity atom inside the nanotube, we use the theoretical results found in reference [4]. Schematic representations of the fully relaxed structures are exemplified in Figure 1. In this figure one can see that, according to the obtained $(6,0)$ SWCNTs relaxed structures, the TM impurities are centered, i.e. their location remain very close to the nanotube axis. On the other hand, according to the $(7,0),(8$, $0),(9,0)$ and $(12,0)$ relaxed structures, it is found that the impurities are displaced from such axis, approaching the internal side of the carbon encapsulation wall. With the exception of the $\mathrm{Mn}$ doped $(7,0)$ nanotube, the impurity atoms are located in an equidistant position relative to the vertices of an hexagon, distant from 1.98 to $2.22 \AA$ from each carbon atom, aligned with the center of such hexagon. It is interesting to point out that the presence of an impurity is always reflected on the carbon neighborhood, where the average $\mathrm{C}-\mathrm{C}$ bond length systematically increases an amount of about $0.02 \AA$. Additionally, it is highly probable to occur electronic charge transfer between the TM and carbon atoms, due to the proximity of such impurity to the tube wall. Therefore, it is possible to infer that the diameter should not be the only relevant

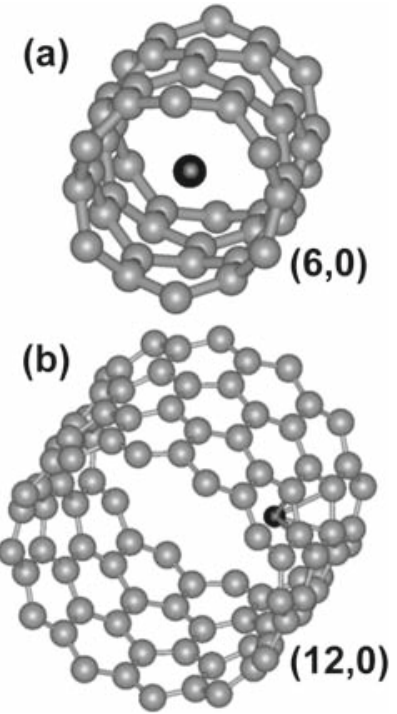

Fig. 1. Position of the metal atom inside the $(6,0)$ and $(12$, $0)$ carbon nanotubes. The black circle corresponds to $\mathrm{Fe}, \mathrm{Ni}$, or $\mathrm{Mn}$ atom, and the grey circles correspond to $\mathrm{C}$ atoms.

Table 1. Calculated binding energies, with $\left(E_{b}^{S P}\right)$ and without $\left(E_{b}^{S U P}\right)$ spin polarization, of $\mathrm{Fe}, \mathrm{Mn}$, and Ni impurities in carbon nanotubes.

\begin{tabular}{cccccccc}
\hline & \multicolumn{3}{c}{$E_{b}^{S P}(\mathrm{eV})$} & & \multicolumn{3}{c}{$E_{b}^{S U P}(\mathrm{eV})$} \\
\cline { 2 - 4 } \cline { 7 - 8 } Nanotube & $\mathrm{Fe}$ & $\mathrm{Mn}$ & $\mathrm{Ni}$ & & $\mathrm{Fe}$ & $\mathrm{Mn}$ & $\mathrm{Ni}$ \\
\hline$(6,0)$ & 3.17 & -0.01 & 1.45 & & 5.54 & 2.80 & 3.90 \\
$(7,0)$ & 1.97 & 0.34 & 0.69 & & 5.21 & 2.22 & 3.00 \\
$(8,0)$ & 1.70 & 0.29 & 0.35 & & 4.92 & 1.70 & 3.00 \\
$(9,0)$ & 1.79 & 0.20 & 0.35 & & 4.93 & 1.70 & 2.90 \\
$(12,0)$ & 1.69 & 0.00 & 0.31 & & 4.70 & 1.70 & 3.10 \\
\hline
\end{tabular}

parameter for the changes on the structure and nanotube properties.

The binding energy, $E_{b}$, is calculated using:

$$
E_{b}=E_{(\text {tube })}+E_{(\text {metal })}-E_{(\text {tube }+ \text { metal })}
$$

where $E_{(\text {tube+metal })}$ is the calculated total energy of the doped tube, $E_{(t u b e)}$ is the total energy of the carbon nanotube and $E_{(\text {metal })}$ is the total energy of an isolated metal atom.

Table 1 shows the calculated binding energies, with and without spin polarization $-E_{b}^{S P}$ and $E_{b}^{S U P}$, respectively, for $\mathrm{Mn}, \mathrm{Fe}$ and $\mathrm{Ni}$ impurities as a function of the tube diameter $D$. The results show that $E_{b}$ decreases with the increasing diameter, i.e. decreasing tube curvature, and saturates at a value which corresponds to a graphene plane interacting with an iron atom. Therefore, it is possible to conclude that highest binding energies of doped nanotubes are the ones with smaller diameters. This is entirely different from non-doped SWCNTs, where the strain energy decreases with the diameter following the classical $1 / D^{2}$ law [22]. Such behavior can be explained by the curvature effect, as mentioned in reference [23], where the binding was found to be stronger at large tube curvatures under radial deformation. It is also possible to observe 


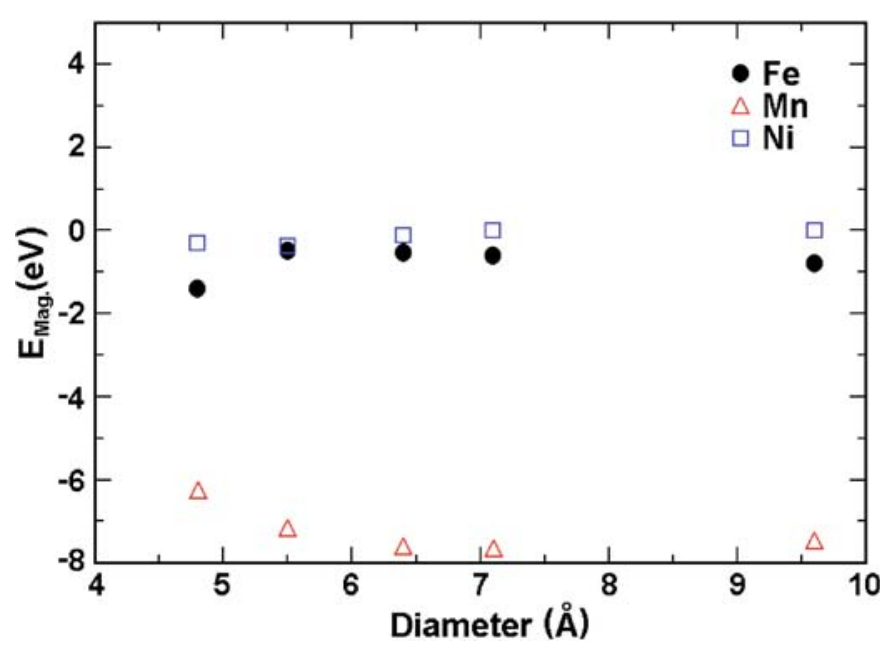

Fig. 2. (Color online) Magnetization energy, $E_{M a g}$, as a function of the nanotube diameter for Fe (full circles), Mn (open triangles), and $\mathrm{Ni}$ (open squares) impurities.

that the metallic impurity which presents the larger binding energy is Fe. Therefore, from the point view of stability, the iron impurity is the most stable one. Such result disagree with the one obtained by Yu-Lian et al. [24], for graphite, where it is reported that the Mn is the most stable impurity. Nevertheless, the present result is supported by the calculation performed by Duffy and coworkers [25], where it is shown that the most favorable position of a manganese atom is above the $\mathrm{C}$ ones, rather than aligned with the hexagon central position.

Additionally, for Mn impurity atoms, which have the smaller number of electron in the $4 d$ orbital when compared to $\mathrm{Ni}$ and $\mathrm{Fe}$, it is found $E_{b}=0$ for the $(12,0)$ tube, using spin polarized calculation. Such behavior can be understood when we compare the resulting $\mathrm{Mn}-\mathrm{C}$ bond length obtained from spin polarized (SP) and spin unpolarized (SUP) calculations. For SP, the average Mn-C bond length, $d_{\mathrm{Mn}-\mathrm{C}}$, is about $2.17 \AA$, while for SUP is $2.84 \AA$. Therefore, it is possible to conclude that for SUP calculation the $\mathrm{Mn}$ atom is not bonding to the carbon nanotube, which leads to such a null value for the bonding energy.

Figure 2 shows the calculated magnetization energy, $E_{\text {mag }}$, obtained from the difference between the total energy of the system with and without spin polarized configurations, plotted against the tube diameter. For Ni, $E_{m a g}$ has the smallest variation, raging from zero to a minimum of $-0.42 \mathrm{eV}$, at $D=5.5 \AA$. For Fe, the magnetization energy reaches a minimum of $-1.41 \mathrm{eV}$, at $D=4.8 \AA$. It increases up to $0.5 \mathrm{eV}$ at $D=5.5 \AA$ and slowly decreases for higher diameters reaching $-0.8 \mathrm{eV}$ at $D=9.6 \AA$. On the other hand, manganese shows a quite different behavior, where $E_{\text {mag }}$ systematically decreases from $-6.33 \mathrm{eV}$ to a saturation value of about $-7.7 \mathrm{eV}$. Such magnetization energy values are the smaller ones when compared with the corresponding results obtained for $\mathrm{Fe}$ and Ni. This result may be related with the peculiar behavior of the
Mn bonding energy, as mentioned above, and the higher magnetization, as described below.

\section{Electronic structure}

Analyzing the calculation results, summarized in Figures 3 and 4 , it is possible to observe that all metal impurities introduce an energy level inside the energy gap region. These figures show that the electronic states inside the gap are predominantly associated to the electrons of the $d$-like orbitals from the metallic atoms, which is consistent with the interpretation that the additional atom (or molecule) represents a defect in a gap material. In other words, it is clear that the gap states are due to the $\mathrm{d}$ and $\mathrm{s}$ orbitals of the impurity atom as one can see in Figures 5 and 6 .

Defining the work function as the difference between the potential energy of an electron in the vacuum level (assumed as zero) and in the highest occupied molecular orbital level on the valence band [26], we obtain the values quoted in Table 2, for nanotubes with and without metal impurities. From such data, it is possible to verify that the work function of SWCNTs varies with the tube diameter from 4.1 to $4.5 \mathrm{eV}$, in agreement with the experimental values $[13,14]$. For doped tubes it is possible to see a significant reduction of the work function in all cases. It is interesting to point out that for $\mathrm{Mn}$ impurities this reduction reaches the maximum value, of about $0.5 \mathrm{eV}$. Therefore, the present calculations indicate that the performance of carbon nanotubes in field emission devices would be sensitive to the incorporation of TM impurities.

\section{Magnetic properties}

An important aspect in the study of carbon nanotubes doped with metal impurities is the investigation of the resulting magnetization and its relation with the tube curvature. Table 2 shows the calculation results obtained for the total magnetic moment, $M$, as a function of the tube diameter. From such results it is possible to observe that $M$ presents a significant variation and rapidly saturates at values corresponding to that ones obtained for graphene planes. For $\mathrm{Fe}$ and Ni impurities, the magnetization decreases for higher diameters. On the other hand, for Mn impurity, the magnetization systematically increases with $D$. Additionally, the smallest values of the total magnetic moment are observed for $\mathrm{Ni}$ and the higher ones for $\mathrm{Mn}$, which are about two times the obtained magnetization for Fe.

Table 3 summarizes the calculation results, obtained using Mulliken population analysis, for the orbital contributions to the magnetic moment of carbon nanotubes containing TM impurities. In each table section $\alpha_{s}, \alpha_{d}$, and $\alpha_{p}$ indicate the corresponding contributions of the $4 s, 3 d$ and $4 p$ electrons of the impurity atom, and $\beta_{p}$ indicates the contribution of the $2 p$ electrons of the adjacent carbon atoms.

The behavior of the magnetic moment for Fe results from the depletion of the $4 s$ orbitals due to confinement 

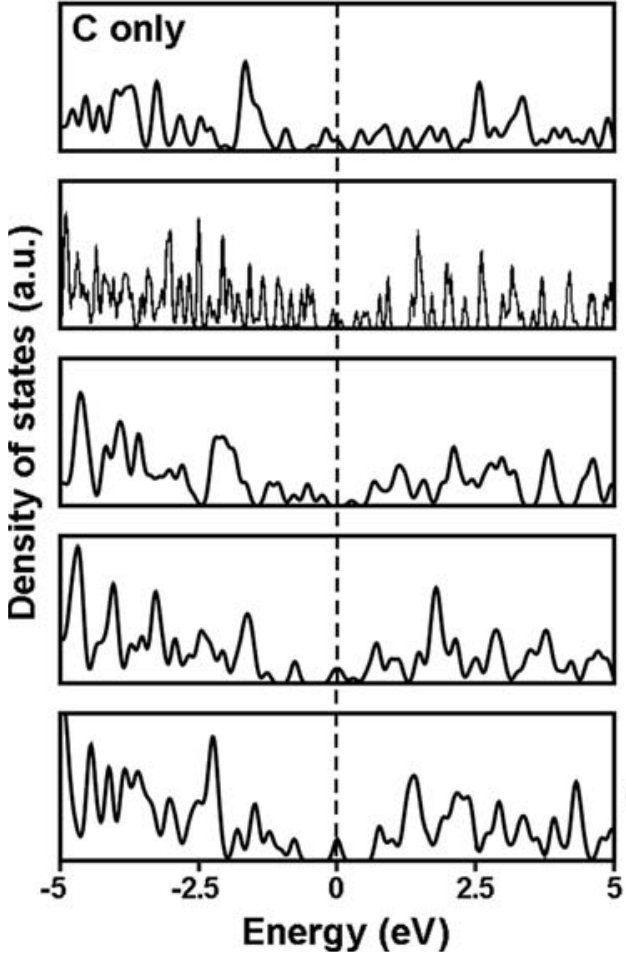

$(6,0)$

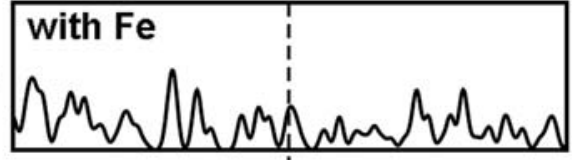

$(7,0)$

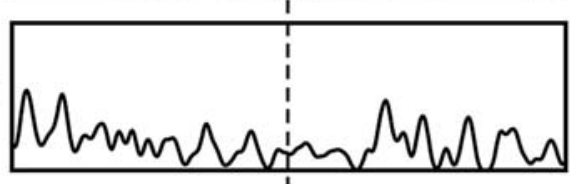

$(8,0)$

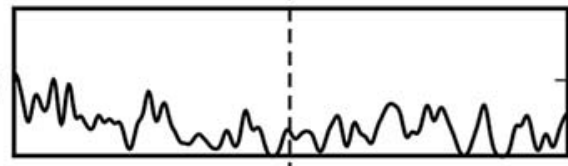

$(9,0)$

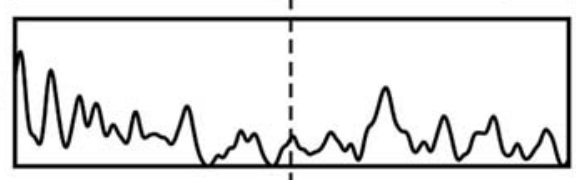

$(12,0)$

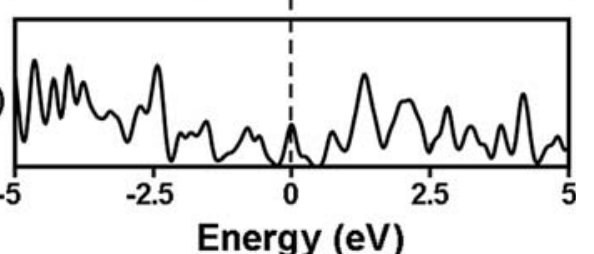

Fig. 3. Density of states of $(6,0),(7,0),(8,0),(9,0)$ and $(12,0)$ carbon nanotubes, with and without Fe impurity atom.
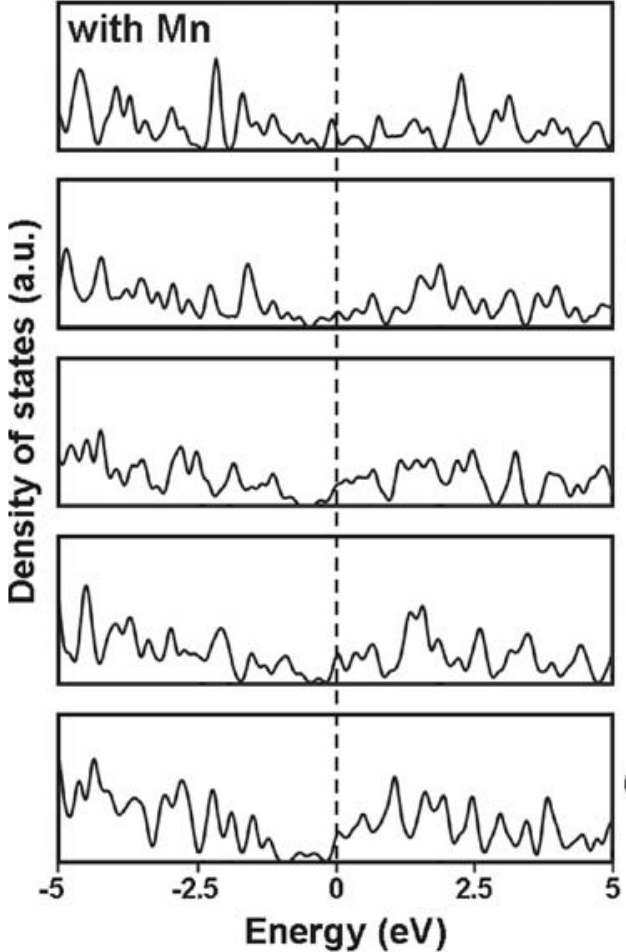

$(6,0)$

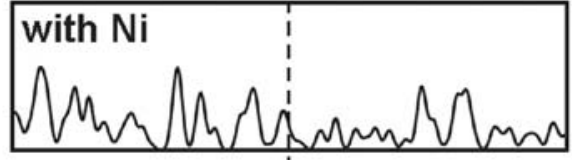

$(7,0)$

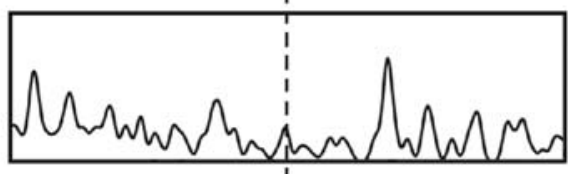

$(8,0)$

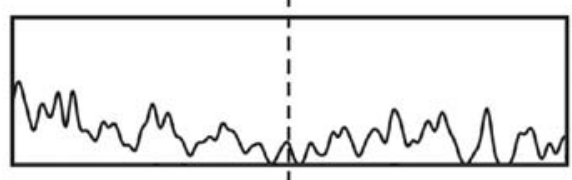

$(9,0)$

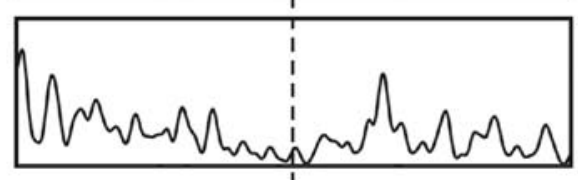

$(12,0)$

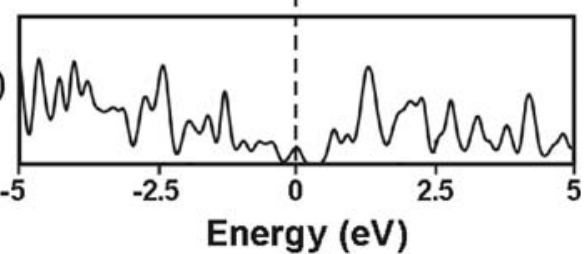

Fig. 4. Density of states of $(6,0),(7,0),(8,0),(9,0)$ and $(12,0)$ carbon nanotubes, with Ni and Mn impurity atoms. 
Table 2. Calculated work function, $W$, and total magnetic moment, $M$, of carbon nanotubes containing Fe, Mn and Ni impurities, as a function of the tube diameter $(D)$. The third column shows the work function of SWCNT's without impurities. The values in brackets indicate the total magnetic moment of an isolated impurity atom.

\begin{tabular}{|c|c|c|c|c|c|c|c|c|}
\hline \multirow[b]{2}{*}{ Nanotube } & \multirow[b]{2}{*}{$\mathrm{D}(\AA)$} & \multicolumn{4}{|c|}{$W(\mathrm{eV})$} & \multicolumn{3}{|c|}{$M\left(\mu_{B}\right)$} \\
\hline & & $\mathrm{C}$ & $\mathrm{Fe}$ & $\mathrm{Ni}$ & $\mathrm{Mn}$ & $\mathrm{Fe}[4.0]$ & $\mathrm{Ni}[2.0]$ & $\operatorname{Mn}[5.0]$ \\
\hline$(6,0)$ & 4.8 & 4.50 & 4.20 & 4.30 & 4.20 & 2.37 & 0.79 & 5.00 \\
\hline$(7,0)$ & 5.5 & 4.50 & 3.95 & 4.12 & 3.84 & 2.55 & 1.18 & 5.62 \\
\hline$(8,0)$ & 6.4 & 4.45 & 3.86 & 3.96 & 3.64 & 2.21 & 1.27 & 5.80 \\
\hline$(9,0)$ & 7.1 & 4.10 & 3.81 & 3.80 & 3.65 & 1.97 & 0.01 & 5.80 \\
\hline$(12,0)$ & 9.6 & 4.10 & 3.91 & 3.92 & 3.56 & 2.00 & 0.03 & 5.90 \\
\hline
\end{tabular}

Table 3. Calculated orbital contributions, in $\mu_{B}$ units, for the resulting magnetic moment of carbon nanotubes containing transition metal impurities obtained using Mulliken population analysis. In each section $\alpha_{s}, \alpha_{d}$, and $\alpha_{p}$ indicate the contributions of the $4 s, 3 d$ and $4 p$ orbitals of the impurity atom, respectively. Additionally, $\beta_{p}$ indicates the contribution of the $2 p$ orbital of the adjacent carbon atoms.

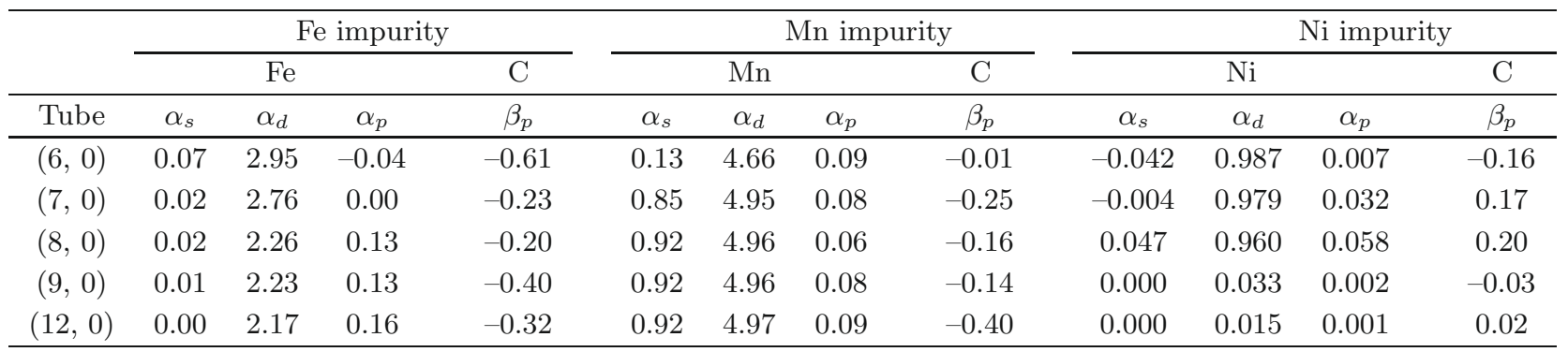
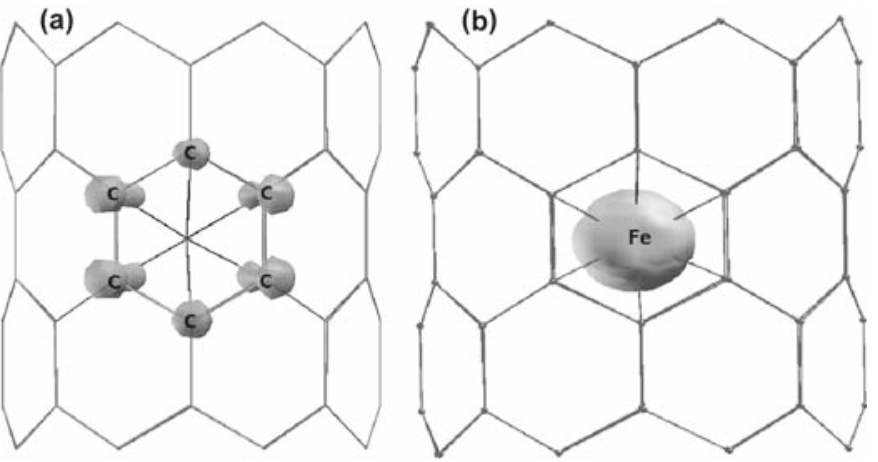

Fig. 5. Spin charge density for Fe atom in a carbon nanotube. (a) Spin-down minus spin-up electronic charge density for a $(8,0)$ cabon nanotube. (b) Spin-up minus spin-down electronic charge density for the same nanotube.

effects. Indeed, from Table 3 data, it is possible to infer about the charge transfer between the $4 s$ and $3 d$ orbitals. For the $(6,0)$ tube, there is charge transfer from the Fe $4 s$ to $\mathrm{Fe} 3 d$ orbitals that leads to an effective $3 d^{6.53} 4 s^{0.33}$ atomic configuration. For the $(7,0),(8,0),(9,0)$, and $(12,0)$ nanotubes, the charge transfer leads to the same $3 d^{6.7} 4 s^{0.44}$ configuration. Nevertheless, according to the results quoted in Table 2, the magnetic moment of such structures presents a significant variation. More careful analysis of the results shows that there is some charge transfer from the $s$ orbital of the iron impurity to the $p$ orbital of the carbon, increasing the net spin. Therefore, it indicates that the curvature effect is the responsible for the decreasing magnetic moment (from $M=2.5$ to $\left.2.0 \mu_{B}\right)$, rather than the confinement effect that leads to electronic charge transfer from the $s$ to the $d$ orbital of the Fe impurity. Figure 5 shows the difference between spin-up and spin-down electronic charge density of an iron impurity atom, where one can see that the majority spin is associated to the $3 d$ orbital.

For $\mathrm{Ni}$ impurity, there is a confinement of $s$ states due to the tube curvature. It results from the electronic charge transfer from the $4 s$ orbitals to $3 d$ and $4 p$ orbitals. Such transference initially increases with the tube diameter, but for the $(9,0)$ and $(12,0)$ tubes it results in the $4 s^{0.48} 3 d^{8.6} 4 p^{0.5}$ configuration for the $\mathrm{Ni}$ atom, and $2 p^{0.5}$ for the adjacent $\mathrm{C}$ atoms, similar to Fe impurities. Such configuration leads to a magnetic moment per spin given by $4 s^{0.24} 3 d^{4.4} 4 p^{0.3}$, resulting in $M=0 \mu_{B}$ for nanotubes with larger diameters.

The calculation results for Mn impurity atoms demonstrate that total magnetic moment systematically increases with the nanotube diameter (see Tab. 2). Such behavior results from the depletion of the $4 s$ orbitals due to confinement, associated with the smaller electronegativity of the manganese. Using the Mulliken population analysis, it is possible to infer that an electron associated to $4 s^{2}$ orbitals migrates to the adjacent carbon atoms rather than to the $3 d$ orbitals, as illustrated in Figure 6, in opposition to the case of $\mathrm{Fe}$ and Ni impurities. Therefore, the small eletronegativity of $\mathrm{Mn}$ associated to the tube curvature induces the transference of electrons from $4 s$ to $3 d$ orbitals of the $\mathrm{Mn}$ atom and $2 p$ orbitals of the adjacent carbon atoms, leading to the final configuration $4 s^{0.92} 3 d^{4.97}$ and to a resulting magnetic moment of about $5.8 \mu_{B}$. 
(a)

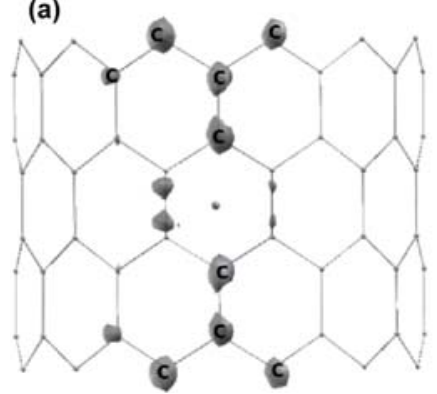

(b)

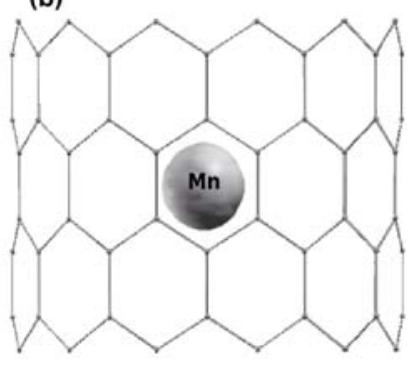

Fig. 6. Spin charge density for $\mathrm{Mn}$ atom in a carbon nanotube. (a) Spin-down minus spin-up electronic charge density for a $(12,0)$ cabon nanotube. (b) Spin-up minus spin-down electronic charge density for the same nanotube.

\section{Conclusions}

In summary, we apply first-principles method to investigate the influence of transition metal impurities on the stability and electronic properties of zigzag carbon nanotubes. The obtained results show that the impurity bind energy decreases with the tube diameter, where iron is the most stable one. The calculations indicate that the performance of carbon nanotubes in field emission devices should be affected by metal impurities, and that the resulting magnetic moment is very sensitive to the impurity element and tube diameter.

This work has been supported by Brazilian agency CNPq and Fundação de Amparo Pesquisa do Estado da Bahia, FAPESB, and INCT - Nanomateriais de Carbono

\section{References}

1. H.W. Kroto, J.R. Heath, S.C. O'Brien, R.F. Curl, R.E. Smalley, Nature (London) 318, 162 (1985)

2. S. Iijima, Nature (London) 354, 56 (1991)

3. N. Hamada, S. Sawada, A. Oshyana, Phys. Rev. Lett. 68, 1579 (1992)

4. S.B. Fagan, R. Mota, A.J.R. da Silva, A. Fazzio, Phys. Rev. B 67, 205414 (2003)

5. Yosuke Yagi, T.M. Briere, M.H.F. Sluiter, V. Kumar, A.A. Farajian, Y. Kawazoe, Phys. Rev. B 69, 075414 (2004)

6. C. Binns, S.H. Baker, A.M. Keen, S.N. Mozley, C. Norris, H.S. Derbyshire, S.C. Bayliss, Phys. Rev. B 53, 7451 (1995)

7. M. Bumer, J. Libuda, H.-J. Freund, Surf. Sci. 327, 321 (1997)

8. N.Y. Jin-Phillipp, M. Rühle, Phys. Rev. B 70, 245421 (2004)

9. Yong-Ju, Jin Choi, Chang-Youn Moon, K.J. Chang, Phys. Rev. B 71, 115441 (2005)

10. M. Weissmann, G. Garcia, M. Kiwi, R. Ramirez, C.C. Fu, Phys. Rev. B 73, 125435 (2006)

11. S. Yuan, Y. Kong, F. Wen, F. Li, Comp. Mater. Sci. 42, 83 (2008)

12. S. Suzuki, C. Bower, O. Zhow, Appl. Phys. Lett. 76, 4007 (2000)

13. M. Shiraishi, M. Ata, Carbon 39, 1913 (2001)

14. Hyun Young Jung, Sung Mi Jung, Lily Kim, Jung Sang Suh, Carbon 46, 969 (2008)

15. P. Ordejon, E. Artacho, J.M. Soler, Phys. Rev. B 53, R10441 (1996)

16. D. Sanchez-Portal, P. Ordejon, E. Artacho, J.M. Soler, Int. J. Quantum Chem. 65, 453 (1997)

17. J.M Soler, E. Artacho, J.D. Gale, A. García, J. Junquera, P. Ordejón, D. Sánchez-Porta, J. Phys.: Condens. Matt. 14, 2745 (2002)

18. W. Kohn, L.J. Sham, Phys. Rev. 140, A1133 (1965)

19. J.P. Perdew, K. Burke, M. Enrzerhof, Phys. Rev. Lett. 77, 3865 (1996)

20. L. Kleinman, D.M. Bylander, Phys. Rev. Lett. 48, 1425 (1982)

21. N. Troullier, J.L. Martins, Phys. Rev. B 43, 1993 (1991)

22. S. Azevedo, R. de Paiva, J.R. Kaschny, J. Phys.: Condens. Matt. 18, 10871 (2006)

23. O. Gulseren, T. Yildirin, S. Ciraci, Phys. Rev. Lett. 87, $116802(2001)$

24. Yu-Liang, Xiao-Hong Yan, Yang Xiao, Nanotechnology 16, $3092(2005)$

25. D.M. Duffy, J.A. Blackman, Phys. Rev. B 58, 7443 (1998)

26. Gang Zhou, Wenhei Duao, Binglin Gu, Phys. Rev. Lett. 87, 095504 (2001) 